\title{
Ultraviolet Illumination Induces Scoparone Production in Kumquat and Orange Fruit and Improves Decay Resistance
}

\author{
Victor Rodov', Shimshon Ben-Yehoshua ${ }^{1}$, Jong Jin Kim', Boris Shapiro, and Yitzhak Ittah ${ }^{2}$ \\ Ministry of Agriculture, ARO Volcani Center, P. 0. Box 6, Bet Dagan 50250, Israel
}

Additional index words. postharvest, phytoalexin, Fortunella margarita, Citrus sinensis

\begin{abstract}
Ultraviolet (UV) illumination $(254 \mathrm{~nm})$ induced production of the phytoalexin scoparone in flavedo of kumquat (Fortunella margarita Lour. Swingle cv. Nagami) and orange [Citrus sinensis (L.) Osbeck cvs. Shamouti and Valencia]. Trace amounts of scoparone $\left(<2.0 \mu \mathrm{g} \cdot \mathrm{g}^{-1}\right.$ fresh weight of flavedo) were detected in nontreated fruits. Phytoalexin accumulation in kumquat reached a peak of $530 \mu \mathrm{g} \cdot \mathrm{g}^{-1} 11$ days after illumination, hut the amount declined rapidly, returning to a trace level 1 month after treatment.. Production of scoparone in illuminated fruits was enhanced by increasing the UV dose from $1.5 \times 10^{3}$ to $9.0 \times 10^{3} \mathrm{~J} \cdot \mathrm{m}^{-2}$ for orange and from $0.2 \times 10^{3}$ to 1.5 $\times 10^{3} \mathrm{~J} \cdot \mathrm{m}^{-2}$ for kumquat and by raising the storage temperature from 2 to $17 \mathrm{C}$. Phytoalexin accumulation correlated with an increase in antifungal activity of flavedo extracts. UV-illuminated kumquat fruit inoculated with Penicillium digitatum Sacc. 2 days after treatment had a lower incidence of decay than the control. Illumination of previously inoculated fruit failed to prevent decay. Kumquat fruit stored at $17 \mathrm{C}$ showed signs of UV-induced peel damage. Chemical name used: 6,7-dimethoxycoumarin (scoparone).
\end{abstract}

Phytoalexins, the low-molecular antimicrobial substances of various chemical structures, are elicited in plant tissues by either biotic (pathogen challenge) or abiotic (wounding, chemicals, irradiation, etc.) (Bailey and Mansfield, 1982) stresses. Shortwave ultraviolet light (UV) is known as a nonspecific phytoalexin elicitor. Since 1971, when Hadwiger and Schwochau described the induction of the isoflavonoid phytoalexin pisatin in UV-treated pea pods, many studies on diverse plants have been devoted to this subject (Beier and Oertli, 1983; Bridge and Klarman, 1973; Cheema and Haard, 1978; Creasy and Coffee, 1988; Kodama et al., 1988; Mercier et al., 1990). Elicitation of phytoalexins by UV illumination increased disease resistance of genetically susceptible genotypes of several species (Andebrhan and Wood, 1980; Bridge and Klarman, 1973; Mercier et al., 1990). Stevens et al. (1990a, 1990b) reported control of postharvest rots by UV illumination of various agricultural commodities, including citrus fruits.

Information on phytoalexin induction by abiotic elicitors in citrus and related fruits has been limited. At the beginning of the 1970s, Riov identified two coumarins in the peel of $\gamma-$ irradiated grapefruit (Citrus paradisi Mcfad.): scopoletin (6methoxy,7-hydroxycoumarin) and scoparone (Riov, 1971; Riov et al., 1971). He associated these compounds with irradiation damage in the peel. Much later, Dubery and Schabort (1987) confirmed these findings and emphasized the antifungal activity of scoparone, as well as that of another irradiation-induced noncoumarin substance (Dubery et al., 1988). A range of coumarinderived antifungal compounds was found by Ben-Yehoshua et al. $(1987 ; 1988)$ in the peel of heat-treated pummelo [Citrus grandis (L.) Osb.] fruit. The concept of scoparone as a citrus

Received for publication 8 Aug. 1991. Accepted for publication 24 Feb. 1992. Contribution 3326-E, 1991 series, from the Volcani Center-ARO. We wholeheartedly thank L. Muszkat, ARti Volcani Center, for doing the GC-MS analy sis, and E. Stern of Tana Industries, Kibbutz Netiv Ha Lamed He, Israel, for providing a dosimetric device and technical information. This work was sup ported by a grant from the Commission of the European Communities STD II, project TS2-CT91-0325. The cost of publishing this paper was defrayed in part by the payment of page charges. Under postal regulations, this paper therefore must be hereby marked advertisement solely to indicate this fact.

'Dept. of Fruit and Vegetable Storage.

${ }^{2}$ Dept. of Food Science. phytoalexin was advanced by Afek et al. (1986) and by Arimoto and Homma (Arimoto et al., 1986, Arimoto and Homma, 1988; Homma and Arimoto, 1988). Recently, Kim et al. (1991) demonstrated that heat treatment of Penicillium-inoculated lemon fruit dramatically enhanced scoparone accumulation and contributed significantly to the development of disease resistance.

Kumquat, a small-fruited relative of the Citrus genus, has not been investigated for its natural antifungal compounds. In kumquat, the composition of flavonoids and terpenoids (groups to which many defense-related substances belong) differs from that of citrus species (Horowitz and Gentili, 1977; Koyasako and Bernhard, 1983). One chemical peculiarity of this fruit is its peel sweetness, noncharacteristic of citrus. The problem of stimulation of endogenous defense mechanisms in kumquat is of special economic importance because export of this exotic fruit is limited by its high susceptibility to decay (mainly that of Penicillium digitatum Sacc.) (Chalutz et al., 1989). The application of fungicides for decay control in kumquat, as proposed by Hall (1986), seems undesirable because the peel of this fruit is consumed along with the pulp.

The subject of this work was the induction of phytoalexin in citrus and kumquat fruit by UV light and its contribution to the improvement of kumquat decay resistance.

\section{Materials and Methods}

Plant material. Mature fruit of 'Nagami' kumquat and 'Shamouti' and 'Valencia' oranges were obtained directly from orchards or packing houses before the application of any postharvest treatment. Fruit samples of uniform size and appearance from one orchard were subjected randomly to various treatments.

$U V$-illumination. Fruit were illuminated either on the day of receipt or (in the case of inoculation experiments) 1 day after receipt. Illumination was carried out at ambient temperature in a chamber with four G15T8 lamps (each of 3.6-W UV output; Tana Industries, Kibbutz Netiv Ha Lamed-He, Israel). Fruit was placed $25 \mathrm{~cm}$ from the UV source. UV dose varied from 0.2 $\times 10^{3}$ to $15 \times 10^{3} \mathrm{~J} \cdot \mathrm{m}^{-2}$ (treatment duration 10 to $750 \mathrm{sec}$, respectively). UV doses were measured by means of a UVX Radiometer (UV Products, Inc., San Gabriel, Calif.). Temperature changes during illumination were negligible and, even 
with the highest UV dose tested, did not exceed 2C. Fruit were transferred to darkness immediately after treatment and stored for 3 weeks at either 2, 11, or 17C. Nontreated fruit stored under the same conditions was used as the control.

Phytoalexin extraction and isolation. Procedures were carried out according to Ben-Yehoshua et al. (1987) and Kim et al. (1991), with slight modifications. Flavedo tissues of orange and kumquat fruit (5 $\mathrm{g}$ fresh weight) were excised with a scalpel and extracted with dichloromethane at a ratio of 1:4 (w/v) for $48 \mathrm{~h}$. The tissue was homogenized in dichloromethane with an Osterizer Cyclotrol blender (Oster, Milwaukee) and the homogenate was filtered in vacuo through Watman No. 1 filter paper. Filtrate was dried over anhydrous magnesium sulfate and concentrated in vacua on a Buchi Rotavapor RE 120 (Buchi, Flawil, Switzerland). The extract was recovered with dichloromethane and dried under nitrogen gas. The dry, crude extracts were kept at $-15 \mathrm{C}$ in vials with $\mathrm{N}$ gas until used.

The separation of crude extracts was carried out by thin layer chromatography (TLC) on silica gel $60 \mathrm{~F}_{254}, 0.2 \mathrm{~mm}$ thick (Merck, Darmstadt, Germany). Plates developed with toluene:ethyl acetate $(1: 1, \mathrm{v} / \mathrm{v})$ were dried and exposed to UV light $(366 \mathrm{~nm})$ for detection of the fluorescent compounds. Individual bands were scraped and extracted with dichloromethane and then concentrated in vacuo.

Phytoalexin analysis. High-performance liquid chromatography (HPLC) was conducted on a Hewlett-Packard HP 1090 Liquid Chromatograph equipped with a 1040 diode-array detector (Hewlett-Packard, Waldbronn, Germany). Octadecyl silane (Merck RP-18, $3.4 \times 250 \mathrm{~mm}, 5-\mu \mathrm{m}$ particles) was used for HPLC separations. A guard column $(1 \mathrm{~cm})$ with the same reversed-phase packing was also used. The isocratic mobile phase was methanol : water $(60: 40)$ at a flow rate of $0.5 \mathrm{ml}-\mathrm{min}$ - $^{\text {. }}$ Peaks were detected at $335 \mathrm{~nm}$. Photodiode array measurements of spectral properties (from 230 to $500 \mathrm{~nm}$ ) for the individual peaks were determined at the apex, base, and slope. The fitting of the three spectra indicated the degree of peak purity.

The structure of phytoalexin was confirmed by its mass spectrum on a Finnigan 5100 gas chromatograph/mass spectrometer (Finnigan, Bremen, Germany) with $\mathrm{Rt}_{\mathrm{x}} 5$ capillary column (dimethyldiphenylpolysiloxane) employing a temperature program of $80 \mathrm{C}$ for $2 \mathrm{~min}$, increasing to $195 \mathrm{C}$ at $25 \mathrm{C} / \mathrm{min}$, and then to $205 \mathrm{C}$ at $0.5 \mathrm{C} / \mathrm{min}$ and holding at $205 \mathrm{C}$ for an additional $5 \mathrm{~min}$. Injector temperature was $250 \mathrm{C}$, ionization energy, $70 \mathrm{eV}$.

A quantitative analysis of scoparone was performed on an Aminco SPF-125 spectrophotofluorometer (excitation at $355 \mathrm{~nm}$, emission at $430 \mathrm{~nm}$; Aminco, Silver Spring, Md.) and on an Hp 1090 Liquid Chromatograph in samples separated from crude extracts on a TLC plate, as described above. Standardization of the analysis was performed with an authentic scoparone sample (Aldrich Chem. Co., Milwaukee). The analysis was repeated in triplicate.

Detection and evaluation of antifungal activity. Methods used were those of Kim et al. (1991). Antifungal activity of the TLC bands was detected directly on the plates with Cladosporium cladosporioides G.A. De Vries as the test organism, according to Dahiya et al. (1984). Samples of crude extracts $(20 \mu \mathrm{l})$ dissolved in dichloromethane ( $\left.5 \mathrm{mg}-\mathrm{ml}-^{-}\right)$were separated by TLC as described above. After air-drying overnight at room temperature, the plate was sprayed with a $C$. cladosporiodes spore suspension in Czapek-Dox medium (106 spores $\left./ \mathrm{ml}^{1}\right)$. The sprayed plate was placed on a humid tray, covered with plastic film and then incubated at $24 \mathrm{C}$ for 2 to 3 days. The presence of antifungal

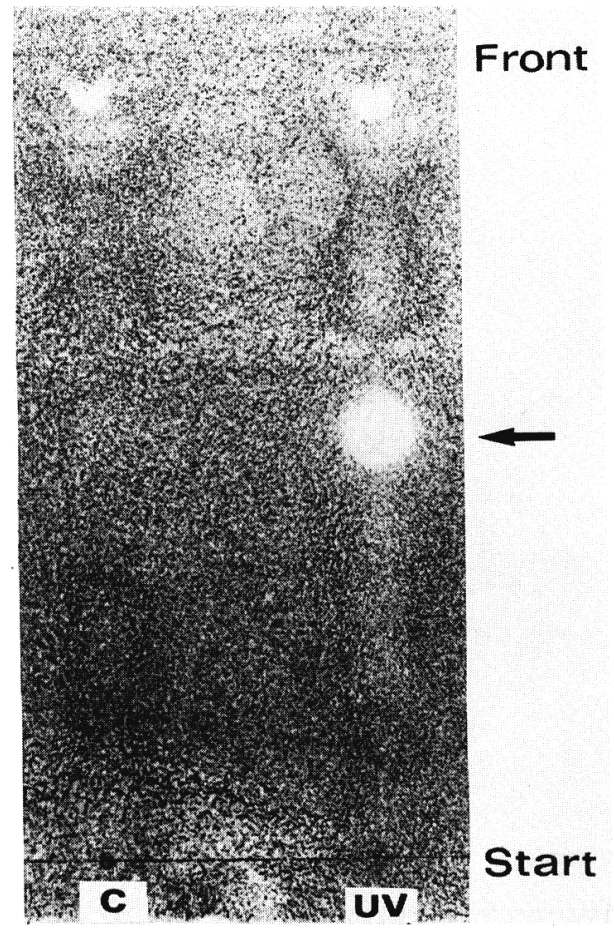

Fig. 1. Detection of the antifungal substance in a crude extract from UV-treated kumquat fruit. Samples: C, extract from nonilluminated fruit; UV, extract from UV-treated fruit (9 days after illumination). Crude extracts were separated by TLC, sprayed with Cladosporium cladosporioides spore suspension, and incubated at 24C for 2 days. The zone of fungal growth inhibition (marked with arrow) indicates the presence of antifungal substance.

activity was indicated by the absence of mycelium around the spot of the compound on the plate.

The degree of antifungal activity of crude extracts was evaluated in a bioassay with $P$. digitatum spores. The crude extracts from $1 \mathrm{~g}$ of flavedo were dissolved in ethanol $(0.2 \mathrm{ml})$, after which $1.8 \mathrm{ml}$ sterile water was added to each. A sample of this solution $(40 \mu \mathrm{l})$ was put into a $35-\mathrm{mm}$ diameter dish (Sterilin Ltd., Feltham, U.K.) containing four wells (11-mm diameter). Each well had $40 \mu$ of $P$. digitatum spore suspension $(2.5 \times$ $10^{4}$ spores $/ \mathrm{ml}$ ) containing $1 \%$ fresh orange juice and $1 \%$ sucrose at $\mathrm{pH}$ 3.7. The prepared dish was placed in a humid tray, covered with plastic film and incubated at $17 \mathrm{C}$ for $20 \mathrm{~h}$.

The percentage of spore germination and germ-tube index were calculated according to Homma and Arimoto (1988). Antifungal activity was estimated as the inhibition of spore germination and germ-tube elongation in comparison to the control (spores germinating in the absence of crude extracts). The assay was repeated in triplicate.

Inoculation of kumquat fruit. Fruit were inoculated on the day of receipt and stored after inoculation at $17 \mathrm{C}$ and $85 \%$ relative humidity. Two methods of fruit inoculation were tested: a) with a spore suspension of $P$. digitatum $\left(10^{5}\right.$ spores $\left./ \mathrm{ml}\right)$ by piercing flavedo with a needle to a depth of 0.7 to $0.8 \mathrm{~mm}$; and b) with dry spores taken from a fruit infected with $P$. digitatum and spread on the intact fruit surface by a cotton tampon. The needle-piercing method was too injurious for the delicate kumquat fruit and the second method, used previously in phytopathological work with kumquat (E. Lomaniec, personal communication), was adopted. About $90 \%$ infection occurred in control 


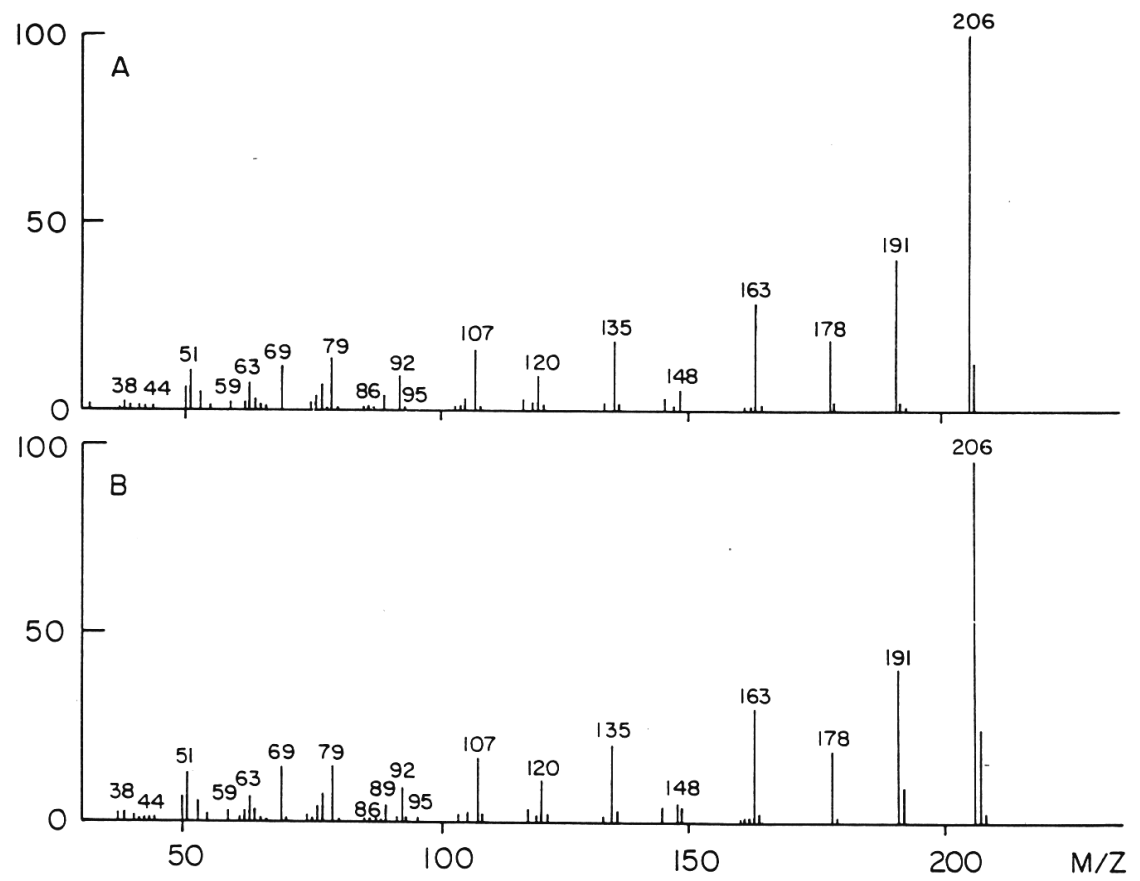

Fig. 2. Mass-spectra of scoparone. Samples: (A) authentic standard (Aldrich); (B) substance extracted from UV-treated fruit. Concentrated dichloromethane extract from $1 \mathrm{~g}$ flavedo was separated by TLC ( 1 toluene : 1 ethylacetate). Band with Rf 0.5 was scraped, eluted with dichloromethane, concentrated and subjected to gas chromatography/mass-spectrometry.

fruit 9 days after inoculation, and $95 \%$ to $100 \%$ infection 14 days after inoculation was obtained.

All experiments were repeated in triplicate and evaluated statistically by Duncan's multiple range test at $P=0.01$.

\section{R e s u l t s}

Isolation and identification of scoparone. UV illumination induced the appearance and accumulation of several fluorescent substances in kumquat and orange. These substances were particularly evident in kumquat fruit, which normally is poor in dichloromethane-extractable fluorescent compounds. One of these substances (Rf 0.5) manifested high antifungal activity in the bioassay with $C$. cladosporoides (Fig. 1). Other induced substances were much less active.

Isolation of the antifungal substance from crude extracts of flavedo by TLC and HPLC and its identification by means of UV, fluorescence, and mass-spectrometry (Fig 2) enabled us to

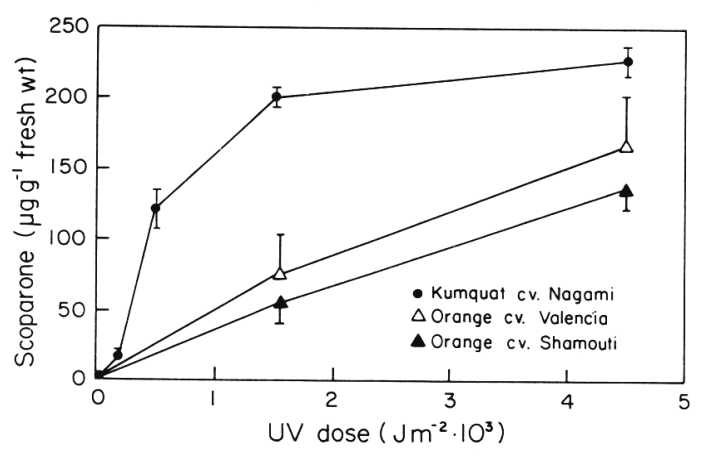

Fig. 3. Effect of UV dose on scoparone accumulation in kumquat and orange fruit. Analysis 6 days after illumination. Scoparone was extracted with dichloromethane, isolated by TLC, eluted, and measured fluorimetrically.

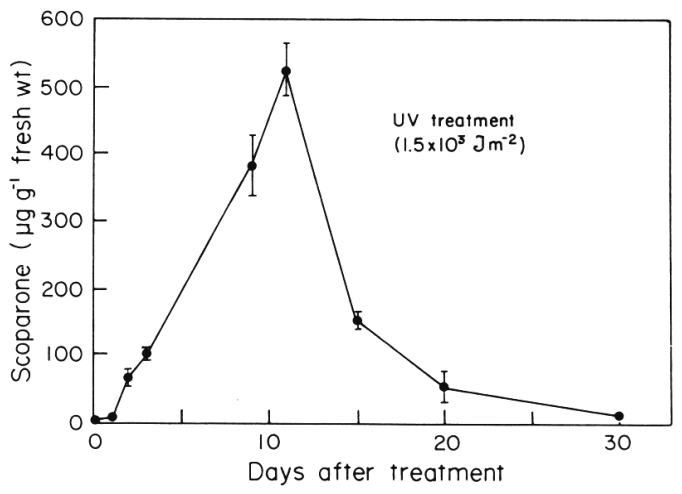

Fig. 4. Time course of scoparone accumulation and depletion in UV-treated kumquat fruit. Extraction and analysis as described in Fig. 2.

identify it as scoparone. According to chromatographic and spectral data, it was found to be identical both to the authentic sample of standard scoparone and to the substance isolated by Kim et al. (1991) from the peel of Penicillium-inoculated and heat-treated lemon [Citrus limon (L.) Burm.f.] fruit. One of the other UV-induced substances was identified as scopoletin on the basis of spectral and chromatographic data.

Scoparone production in fruit. Kumquat and orange both contained trace amounts of scoparone in flavedo of nonilluminated fruit (up to 1.8 and $0.6 \mu \mathrm{g} \cdot \mathrm{g}^{-1}$, respectively). During storage (1 to 3 months at 17C), the quantity of scoparone in nontreated fruits increased slightly, but did not exceed $4.5 \mu \mathrm{g} \cdot \mathrm{g}^{-1}$ fresh weight. UV illumination stimulated scoparone production in both species. Oranges treated with a UV dose of $1.5 \times 10^{3} \mathrm{~J} \cdot \mathrm{m}^{-2}$ and stored for 6 days after treatment at $17 \mathrm{C}$, contained 50 to 70 $\mu \mathrm{g} \cdot \mathrm{g}^{-1}$ of scoparone, while kumquat fruit contained $>200 \mu \mathrm{g} \cdot \mathrm{g}^{-1}$ 